\title{
Very high energy gamma-ray production inside the massive binary system Cyg X-1/HDE 226868
}

\author{
W. Bednarek ${ }^{1}$ and F. Giovannelli ${ }^{2}$ \\ 1 Department of Experimental Physics, University of Łódź, 90-236 Łódź, ul. Pomorska 149/153, Poland \\ e-mail: bednar@fizwe4.fic.uni.lodz.pl \\ 2 INAF - Istituto di Astrofisica Spaziale e Fisica Cosmica, Sezione di Roma, Area di Ricerca CNR di Roma-2, \\ via Fosso del Cavaliere 100, 00133 Roma, Italy \\ e-mail: franco.giovannelli@iasf-roma.inaf.it
}

Received 30 June 2006 / Accepted 6 December 2006

ABSTRACT

\begin{abstract}
$\mathrm{TeV} \gamma$-ray emission has recently been discovered by Cherenkov telescopes from two microquasars, LS 5039 and LS I $+61^{\circ} 303$. This emission is likely to be produced inside the binary system since in both cases variability with the orbital period of the binary has been discovered. In fact, such emission features have recently been predicted by the inverse Compton (IC) $\mathrm{e}^{ \pm}$pair cascade model. In this model, electrons accelerated in the jet develop a cascade in the anisotropic radiation of the massive star. The $\gamma$-ray spectra emerging from the cascade strongly depends on the location of the observer with respect to the orbital plane of the binary. Here we apply this model to investigate the possible $\gamma$-ray emission features from another compact massive binary of the microquasar type, Cyg X-1. We conclude that the observational constraints at lower energies (from $\mathrm{MeV}$ to $\mathrm{GeV}$ ) suggest that the spectrum of electrons injected in the jet is likely steeper than in other $\mathrm{TeV} \gamma$-ray microquasars. The cascade $\gamma$-ray spectrum produced by electrons with such a spectrum in Cyg X-1 should be below the sensitivities of the MAGIC and VERITAS class Cherenkov telescopes. However, if the electron spectrum is flatter, then the highest $\mathrm{TeV} \gamma$-ray fluxes are predicted at $\sim 7 \mathrm{~h}$ before and after the phase when the compact object is in front of the massive star. We suggest that Cherenkov telescopes should concentrate on these ranges of phases since the TeV flux can vary by a factor of $\sim 20$ with the period of the Cyg X-1 binary system. Moreover, the model predicts clear anticorrelation of the $\mathrm{GeV}$ and $\mathrm{TeV} \gamma$-ray emission. This feature can be tested by the future multiwavelength observations with the AGILE and GLAST telescopes in the GeV energy range and the MAGIC and VERITAS telescopes in the TeV energy range.
\end{abstract}

Key words. gamma-rays: theory - radiation mechanisms: non-thermal - stars: binaries: close - stars: individual: Cyg X-1

\section{Introduction}

The importance of high energy processes in X-ray binary systems has been hypothesized for about two decades and in fact some evidence (although not statistically significant) has been reported in the literature (for review see, e.g., Weekes 1988, 1992). However, only one of these early Cherenkov observations of Cen X-3 has been later confirmed, and remains unquestioned up to the present time (Chadwick et al. 2000; Atoyan et al. 2002). Some of these well-known binary systems have been also related to the EGRET sources above $100 \mathrm{MeV}$ due to directional coincidence since they are close to or inside of relatively large error boxes of some EGRET sources: LS I +61 $303 \equiv 2$ CG135+01 $\equiv$ 2EG J0241+6119 (Thompson et al. 1995; Kniffen et al. 1997), Cyg X-3 $\equiv 2$ EG J2033-4112 (Mori et al. 1997), Cen X-3 (Vestrand et al. 1997), and LS 5039 $\equiv$ 3EG J1824-1514 (Paredes et al. 2000). However, only very recently some binary systems belonging to the class of high mass binaries have been explicitly detected at high energy $\gamma$-rays by Cherenkov telescopes. The HESS collaboration reported the detection of TeV $\gamma$-ray emission from the Be type binary system PSR B1259-63, close to the periastron passage (Aharonian et al. 2005a). Also $\mathrm{TeV} \gamma$-rays have been detected from LS 5039 (HESS collaboration, Aharonian et al. 2005b; Aharonian et al. 2006a) and LS I +61 303 (MAGIC collaboration, Albert et al. 2006). These two objects belong to the class of the so-called microquasars. These objects show evidence of anisotropic outflows usually interpreted as jet structures similar to the relativistic jets observed in active galactic nuclei. Such jets in microqusars might be created in the inner parts of the accretion disks around compact objects such as a neutron or a solar mass black hole that is accreting matter from the companion star.

In fact, microquasar features, very similar to those observed in LS 5039 and LS I +61 303, are also observed in the wellknown object, Cyg X-1. It has been detected by the COMPTEL instrument up to $\sim 10 \mathrm{MeV}$ with a spectrum very close to a power law with differential spectral index 2.6 (McConnell et al. 2000, 2002). However, the source has not been observed at higher energies by the EGRET instrument (see the upper limit in McConnell et al. 2000). The MeV $\gamma$-ray emission is usually interpreted in terms of two component thermal and nonthermal comptonization models from the accretion disk (e.g. Gierliński et al. 1999), or as a result of comptonization of the stellar photons by relativistic electrons inside the jet (Bednarek et al. 1990; Georganopoulos et al. 2002; Romero et al. 2002). The general picture of the production of $\gamma$-rays is that they arise as the result of comptonization of stellar photons by electrons in the jet. This model has recently been applied to the microquasar LS 5039 (Dermer \& Böttcher 2006; Bosch-Ramon et al. 2006). However, these models do not take into account Inverse Compton (IC) $\mathrm{e}^{ \pm}$pair cascade processes. Cascade processes become important for these binary systems since the optical depth for electrons (which are isotropic in the jet) depends largely on 
their temporary directions (for detailed calculations of the optical depths, see Bednarek 1997, 2000, and more recently, e.g., Sierpowska \& Bednarek 2005; Böttcher \& Dermer 2005; Dubus 2006; Bednarek 2006a). Due to this effect, electrons lose energy mainly in directions for which the optical depths are the largest, but not in the direction toward the observer (see the previously cited papers). The calculations of the $\gamma$-ray spectra, which are produced in the anisotropic IC $\mathrm{e}^{ \pm}$pair cascade toward the observer, and their light curves as a function of the phase of the binary system, have recently been calculated by (Bednarek 2006a). These calculations are based on the previous work done for other compact binary systems such as Cyg X-3 (Bednarek 1997), and Cen X-3 (Bednarek 2000; Bednarek \& Giovannelli 1999). Recently, the cascade model for the high energy processes inside compact massive binaries has been successfully applied to the binary system LS I +61 303, predicting the phases at which the maximum $\mathrm{TeV} \gamma$-ray emission can be expected (Bednarek 2006a,b). Such a prediction was confirmed by MAGIC observations (see Albert et al. 2006). As an alternative scenario, hadronic production of $\gamma$-rays and neutrinos from these binaries has been also considered (e.g. Romero et al. 2005; Aharonian et al. 2006b). In view of the recent discoveries of the TeV $\gamma$-ray emission from microquasars LS 5039 and LS I +61 303, we consider the IC $\mathrm{e}^{ \pm}$pair cascade model for possible high energy processes in another binary system, Cyg X-1/HDE 226868.

\section{The binary system Cyg X-1/HDE 226868}

Cygnus X-1 has been the most extensively studied stellar mass black hole candidate since its discovery as one of the most intense X-ray sources in the sky (Bowyer et al. 1965). In the Xrays the source shows two emission states (see, e.g., Priedhorsky et al. 1983; Ling et al. 1983; Liang \& Nolan 1983), spending most of the time $(\sim 90 \%)$ in the so-called hard state (low level of soft X-ray emission) and the remaining time in the soft state. Cyg X-1 was also identified as a radio source by Braes \& Miley (1971). High resolution radio observations show a steady jet from this source in the hard X-ray spectral state (Stirling et al. 2001) and also a radio and optical lobe oriented along the jet direction (Gallo et al. 2005). The jet appears when the X-ray spectrum starts softening, and its intrinsic velocity is estimated on $\geq 0.3 \mathrm{c}$ (Fender et al. 2006).

Hjellming \& Wade (1971) identified Cyg X-1 with the optical star HDE 226868, which is an O9.7 Iab type massive star inside a binary system with a period of 5.6 days (Webster \& Murdin 1972; Bolton 1972). The main parameters of this binary system have been recently confirmed by Ziółkowski (2005): the mass of the optical companion $M_{\star}=40 \pm 10 M_{\odot}$; its radius $R_{\star}=22 \pm 3 R_{\odot}$; an effective temperature of $T_{\star}=30000 \pm 2000 \mathrm{~K}$ for a distance to the system $D=1.8-2.35 \mathrm{Kpc}$; and a mass of the compact object $M_{\mathrm{x}}=16 \pm 3 M_{\odot}$, which is in an almost circular orbit $e=0$, with an inclination angle of the binary $i=33^{\circ} \pm 5^{\circ}$. In this paper we adopt the values for the binary system shown in Table 1.

\section{A microquasar in the compact massive binary}

We consider a generally accepted scenario for microquasars, in which electrons are accelerated continuously along the jet. The jet propagates from the compact object along an axis perpendicular to the plane of the binary system. The details of such a general scenario have already been described in Bednarek (2006b).
Table 1. Parameters of the binary system and the jet.

\begin{tabular}{cc}
\hline \hline Parameter & Value \\
\hline$R_{\star}:$ stellar radius & $20 R_{\odot}$ \\
$T_{\star}:$ surface temperature & $3 \times 10^{4} \mathrm{~K}$ \\
$M_{\star}:$ stellar mass & $40 \pm 10 M_{\odot}$ \\
$M_{\star}:$ companion mass & $16 \pm 3 M_{\odot}$ \\
$a:$ orbital radius & $2.15 R_{\star}$ \\
$e:$ eccentricity & 0 \\
$i:$ inclination & $30^{\circ}$ \\
\hline$r_{\text {in }}:$ disk inner radius, radius of the jet & $10^{7} \mathrm{~cm}$ \\
$\alpha:$ jet opening angle & $0.1 \mathrm{rad}$ \\
$z_{\min }:$ inner boundary of the active jet & $0.1 R_{\star}$ \\
$z_{\max }:$ outer boundary of the active jet & $10 R_{\star}$ \\
$\eta:$ magnetization parameter of the jet & $1.0-0.01$ \\
$B_{\mathrm{d}}:$ magnetic field at the base of the jet (for $\left.\eta=1\right)$ & $10^{5} \mathrm{G}$ \\
$\xi:$ acceleration efficiency of electrons & $0.5-0.01$ \\
\hline
\end{tabular}

Here we repeat the basic points of the scenario for the sake of completeness. The shock acceleration mechanism is usually accepted as being responsible for the acceleration of the electrons. It is assumed that electrons are injected with the power-law spectrum extending up to the maximum energies determined by the balance between the acceleration rate and the most efficient energy loss rate. In the case of microquasars, the synchrotron process (determined by the magnetic field strength locally in the jet) or the inverse Compton process (determined by the radiation field of the massive star) usually provide the most efficient cooling for the electrons.

The magnetic field strength inside the jet can be estimated on the basis of the equipartition arguments concerning the relation between the accretion disk luminosity and the kinetic power of the jet, i.e., the so called jet-accretion-disk symbiosis model developed by Falcke et al. (1995) and Falcke et al. (2004). In fact, in the case of Cyg X-1, the X-ray luminosity (probably produced in the accretion disk) and the jet kinetic power are estimated to be of the same order (Gallo et al. 2005). Note, however, that this is not always the case since some other binaries are clearly underluminous, e.g., LS 5039 and LS I +61 303. Moreover, it is commonly expected, on the basis of the equipartition arguments, that the energy density of the magnetic field inside the jet should be related to the kinetic power of the jet (see, e.g., Bosch Ramon et al. 2005). Therefore, we accept the hypothesis that the magnetic field energy density in the inner part of the accretion disk (at the base of the jet) should be related to the energy density of the disk radiation (see also, e.g., Bednarek 2005). On the basis of these arguments we arrive to the conclusion that the magnetic field in the jet can be as large as $B_{\mathrm{d}} \sim 10^{5} \mathrm{G}$ (e.g., Bednarek 2006b). Assuming a simple conical structure for the jet (fixed opening angle $\alpha$ ), the magnetic field in the jet should drop with the distance from its base according to

$B(x)=B_{\mathrm{d}} \eta /(1+\alpha z) \approx B_{\mathrm{d}} \eta /(\alpha z)$ for $z \gg 1 / \alpha$,

where $\alpha$ is assumed to be of the order of $0.1 \mathrm{rad}$, and $\eta$ is the ratio of the energy density of the magnetic field to the energy density of the disk radiation at the base of the jet (magnetization parameter of the jet). This parameter describes how far the magnetic field is from the equipartition with respect to the kinetic energy density of the jet plasma. $z$ is the distance along the jet from its base expressed in units of the disk inner radius $r_{\text {in }}$, where $r_{\text {in }}$ is of the order of $10^{7} \mathrm{~cm}$ for the compact object with the mass of the order of $10 M_{\odot}$. 
Having defined the magnetic field strength at different parts of the jet, we can estimate the acceleration rate of the electrons (with energy $E$ and the Lorentz factors $\gamma$ ),

$\dot{P}_{\mathrm{acc}}(\gamma)=\xi c E / r_{\mathrm{L}} \approx 10^{13} \xi B \mathrm{eV} \mathrm{s}^{-1}$,

where $\xi$ is the acceleration efficiency, $r_{\mathrm{L}}=E / e B$ is their Larmor radius, $B$ is the magnetic field at the acceleration site (in Gauss), $e$ is the electron charge, and $c$ is the velocity of light. In Eq. (1), a fundamental role is played by the acceleration efficiency of particles $\xi$, but this is in fact unknown. There are some observational suggestions that $\xi$ should be not far from unity in the case of relativistic shocks, e.g. the presence of relativistic leptons with energies $\sim 10^{15} \mathrm{eV}$ inside the Crab Nebula. On the other hand, the theory of shock acceleration processes estimates $\xi$ as $\sim 0.1(v / c)^{2}$, i.e., $>0.01$ for $v>0.3 c$ (Malkov \& Drury 2001). Therefore, in this paper we consider the values of the acceleration efficiency of electrons in the jet in the range $\xi=0.5-0.01$. The basic parameters that describe the jet are shown in Table 1 .

As we noted above, during the acceleration process, electrons lose energy mainly by the synchrotron and the inverse Compton (IC) processes. We are interested in situations in which electrons can be accelerated to $\mathrm{TeV}$ energies. This is not easy to obtain relatively close to the base of the jet where the magnetic field is large and the synchrotron losses very strong. Therefore, since we are interested in efficient injection of high energy IC $\gamma$-rays by electrons in the jet, it makes sense to consider distances from the accretion disk for which the IC energy losses on the massive star radiation are at least comparable to synchrotron losses. In this cascade model we will concentrate on the parameter range adequate for the production of $\mathrm{TeV} \gamma$-rays.

Let us estimate the maximum energies of electrons in the shock acceleration process allowed by the energy losses. In these estimations we concentrate on the parts of the jet that are above $z_{\text {min }}=0.1 R_{\odot}=1.4 \times 10^{11} \mathrm{~cm}$, since very close to the base of the jet the radiation field from the accretion disk (and disk corona) may dominate over the massive star radiation field (total luminosity of the disk is estimated in several times $10^{37} \mathrm{erg} \mathrm{s}^{-1}$, see, e.g., Poutanen et al. 1997). At $z_{\text {min }}$, the disk radiation field is diluted by the factor of $\sim\left(\beta z_{\min } / r_{\text {in }}\right)^{2} \approx 10^{-6}-10^{-4}$, where $r_{\text {in }} \sim$ $10^{7} \mathrm{~cm}$ is the inner radius of the disk around the $10 M_{\odot}$ black hole, and the parameter $\beta=r_{\text {in }} / r_{\text {cor }} \approx 0.01-0.1$ defines the extent of the disk corona assumed in the range of $r_{\text {cor }} \approx 10-100 r_{\text {in }}$. Note that the massive star luminosity in Cyg X-1 is clearly larger than the disk luminosity. Therefore, disk radiation can become important only in a relatively small part of the inner jet.

The energy loss rate by the synchrotron process is given by,

$\dot{P}_{\text {syn }}(\gamma)=\frac{4}{3} \pi \sigma_{\mathrm{T}} c U_{\mathrm{B}} \gamma^{2} \approx 2.7 \times 10^{-3} B^{2} \gamma^{2} \quad \mathrm{eV} \mathrm{s}^{-1}$,

where $U_{\mathrm{B}}=B^{2} / 8 \pi$ is the energy density of the magnetic field, $\gamma$ is the Lorentz factor of the electrons, and $\sigma_{\mathrm{T}}$ is the Thomson cross section. Electrons lose energy on the IC process in the Thomson ( $\mathrm{T}$ ) regime, if their Lorentz factors are,

$\gamma \ll \gamma_{\mathrm{T} / \mathrm{kN}}=m c^{2} / 3 k_{\mathrm{B}} T \approx 2 \times 10^{5} / T_{4}$,

(where $T=10^{4} T_{4} \mathrm{~K}$ is the surface temperature of the massive star that defines the black body spectrum, and $k_{\mathrm{B}}$ is the Boltzman constant), and in the Klein-Nishina (KN) regime, for the Lorentz factors $\gamma \gg \gamma_{\mathrm{T} / \mathrm{KN}}$. The energy loss rate in the Thomson regime is given by

$\dot{P}_{\mathrm{IC}}^{\mathrm{T}}(\gamma)=\frac{4}{3} \pi \sigma_{\mathrm{T}} c U_{\mathrm{rad}} \gamma^{2} \approx 3.8 T_{4}^{4} \gamma^{2} / r^{2} \mathrm{eV} \mathrm{s}^{-1}$, where $U_{\text {rad }}=4.7 \times 10^{13} T_{4}^{4} / r^{2} \mathrm{eV} \mathrm{cm}^{-3}$, and $r$ is the distance to the center of the massive star in units of its radius $R_{\star}$. The energy loss rate in the $\mathrm{KN}$ regime depends only logarithmically on the Lorentz factor of the electrons. We approximate these losses by (see, e.g., Blumenthal \& Gould 1970),

$\dot{P}_{\mathrm{IC}}^{\mathrm{KN}}(\gamma) \approx \dot{P}_{\mathrm{IC}}^{\mathrm{T}}\left(\gamma_{\mathrm{T} / \mathrm{KN}}\right) \ln \left(4 k_{\mathrm{B}} T \gamma / m c^{2}-2\right)$.

The maximum energies of the accelerated electrons are determined by the balance between the acceleration mechanism and by the most efficient mechanism for the energy losses. The comparison of Eqs. (2) and (3) gives the maximum Lorentz factors of the electrons due to the synchrotron energy losses allowed,

$\gamma_{\mathrm{syn}} \approx 6 \times 10^{7}(\xi / B)^{1 / 2}$.

The maximum energies allowed by the IC process in the Thomson regime are (from Eqs. (2) and (5)),

$\gamma_{\mathrm{IC}}^{\mathrm{T}} \approx 1.6 \times 10^{6}(\xi B)^{1 / 2} r / T_{4}^{2}$,

and in the $\mathrm{KN}$ regime are (from Eqs. (2) and (6)),

$\gamma_{\mathrm{IC}}^{\mathrm{KN}} \approx 1.5 \times 10^{5}\left(2+\mathrm{e}^{\left(71 \xi B r^{2} / T_{4}^{2}\right)}\right) / T_{4}$.

Note that the limit given by Eq. (8) is only valid provided that $\gamma_{\mathrm{IC}}^{\mathrm{T}}<\gamma_{\mathrm{T} / \mathrm{KN}}$, i.e., when the parameters of the jet and the massive star fulfill the following condition, $T_{4} / r>8(\xi B)^{0.5}$ (from Eqs. (4) and (8)). In any other case, the limit given by Eq. (9) has to be taken.

The Larmor radii of electrons accelerated to the maximum Lorentz factors allowed by the radiation constraints has to be smaller than expected from the constraints imposed by the size of the jet. The Larmor radius of electrons (defined below Eq. (2)) is thus lower than the size of the jet, $r_{\mathrm{L}}<(1+\alpha z) r_{\text {in }}$ (see above), provided that $\gamma_{\text {size }}<e B_{\mathrm{d}} \eta / m_{\mathrm{e}}$. For the considered parameters and the applied model for the magnetic field distribution along the jet (see Eq. (1)), $\gamma_{\text {size }}<6 \times 10^{8}$. Note that this limit is independent of the distance from the base of the jet. It does not impose additional constraints on the values obtained above for distances from the base of the jet that are of interest in this model.

The maximum Lorentz factors to which electrons can be accelerated at a specific part of the jet are determined by the acceleration efficiency and the energy losses through synchrotron and IC processes. These energy loss processes depend on the conditions in the jet (i.e., magnetic field strength and acceleration efficiency), on the parameters of the massive star (its radius and surface temperature), and on the distance of the acceleration region from the massive star. In Fig. 1, we plot the maximum Lorentz factors of electrons as a function of the distance from the base of the jet (given by either Eqs. (7), (8), or (9)), for selected values of the acceleration efficiency, $\xi$, and the ratio, $\eta$. The maximum energies of the accelerated electrons increase with the distance from the base of the jet as $\propto z^{1 / 2}$, when the acceleration process is saturated by synchrotron energy losses (see also Bosch Ramon et al. 2006). However, in the regions of the jet where the magnetic field is relatively weak (farther from the base of the jet but still close to the massive star), the acceleration process is saturated by IC energy losses. Then, the maximum Lorentz factors of the electrons are significantly lower (note the broad parabolic dips in Fig. 1, especially evident during the periastron passage of the compact object).

In a previous work (Bednarek 2006b), we have shown that electrons accelerated in the jet have to cool locally, i.e., close the the acceleration site, due to the very large energy losses, provided that they are injected within $\sim 100 R_{\star}$ from the base of the 

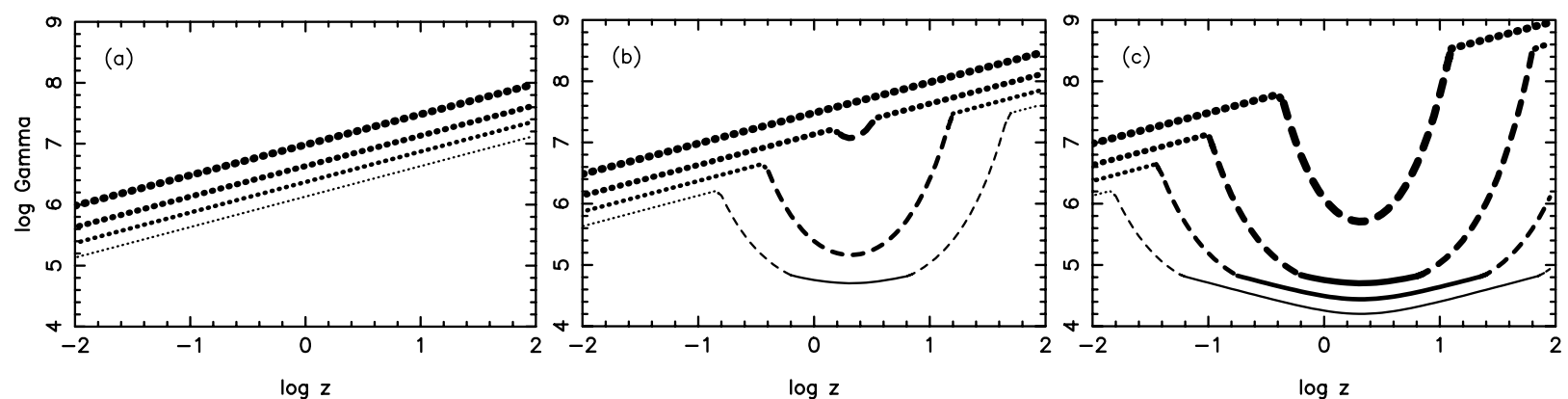

Fig. 1. The maximum Lorentz factors of electrons as a function of the distance, $z$ from the base of the jet (measured in units of the stellar radius $R_{\star}$ ) for different acceleration efficiencies: $\xi=0.5$ (from the upper, thickest curve), $0.1,0.03$, and 0.01 (to the bottom, finest curve) and two ratios of the magnetic field to disk radiation energy densities at the base of the jet equal to $\eta=1 \mathbf{a}$ ), $0.1 \mathbf{b}$ ), and $0.01 \mathbf{c}$ ). The Maximum Lorentz factors are obtained from the comparison of the acceleration rate with the energy loss rate on synchrotron process (dotted lines), inverse Compton process on scattering of stellar radiation from the massive star HDE 226868 in the Thomson regime (solid curve), and the Klein-Nishina regime (dashed curves). The base of the jet is at the distance of $2.15 R_{\star}$ from the center of the massive star.
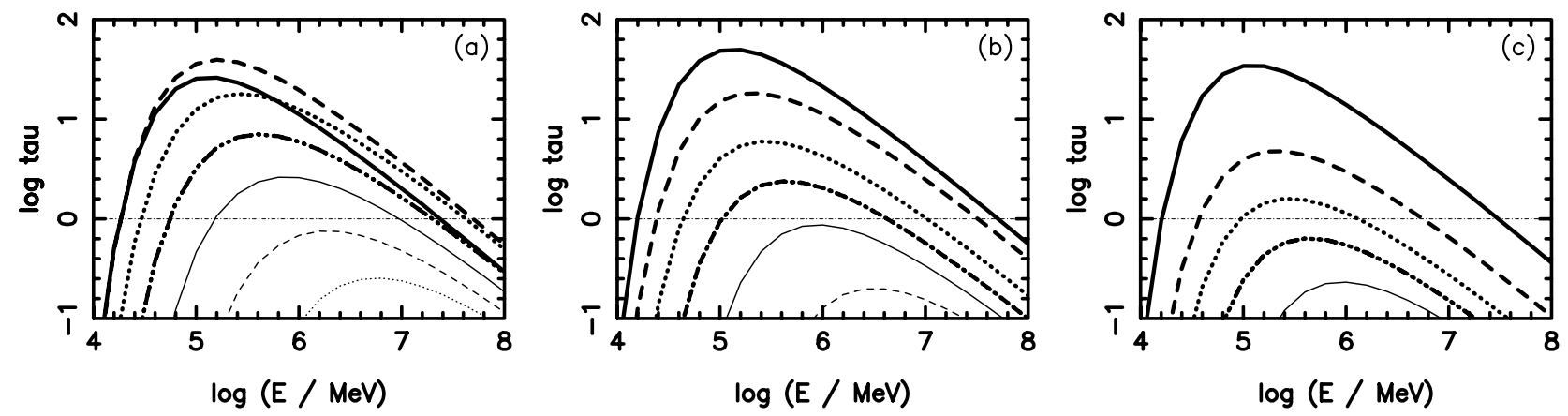

Fig. 2. a) The optical depths for $\gamma$-rays (as a function of their energy) on $\mathrm{e}^{ \pm}$pair production in collisions with stellar photons calculated for $\gamma$-rays propagating from a specific distance from the massive star up to the infinity. $\gamma$-rays are injected at three different distances from the massive star HDE 226868, $\left.z=2 R_{\star} \mathbf{a}\right), z=5 R_{\star} \mathbf{b}$ ), and $z=20 R_{\star} \mathbf{c}$ ), and for selected angles measured from the direction defined by the centers of the stars, $\alpha=0^{\circ}$ (i.e., toward the center of the massive star the optical depth is calculated only to the surface of the star, thick solid curve), $30^{\circ}$ (thick dashed), $60^{\circ}$ (thick dotted), $\alpha=90^{\circ}$ (thick dot-dashed), $120^{\circ}$ (thin solid), $150^{\circ}$ (thin dashed), and $180^{\circ}$ (thin dotted).

jet. At larger distances accelerated electrons are advected outside the binary system (along the jet) and can produce high energy radiation through other processes. However, in such a case, we do not expect any clear variability for the $\gamma$-ray emission with the period of the binary system. Therefore, we do not consider that possibility in this paper.

Following our previous work, we also assume that electrons are injected with the power-law spectrum described by the single spectral index, independent of the distance from the base of the jet $z$ (typical values from the range 2-2.6 are considered). However, the injection rate of electrons, i.e., the power transfered from the magnetized plasma to relativistic electrons $L_{\mathrm{e}}$, can differ, depending on a range of possible processes occurring in the jet. Two different likely scenarios are considered:

1. constant injection of electrons along the jet from its base at $z_{\min }: L_{\mathrm{e}}=$ const.,

2. injection rate drops along the jet according to $L_{\mathrm{e}}(z) \propto z^{-2}$.

\subsection{Propagation of $\gamma$-rays}

Since the Cyg X-1 binary system is very compact (with a separation of the stars of only $a=2.15 R_{\star}$ ), $\gamma$-rays produced in the part of the jet that is closer than a few tens of $R_{\star}$ from the massive star can be efficiently absorbed by collisions with the thermal radiation from the massive star. Following our previous works (Bednarek 1997, 2000; Sierpowska \& Bednarek 2005), we calculate the optical depths for $\gamma$-rays as a function of the energy in the most general case, i.e., for an arbitrary distance from the massive star and direction of their propagation (for an angle measured from the direction defined by the centers of the stars). The results are shown in Fig. 2 for an injection distance of $\gamma$-rays from the center of the massive star at $2 R_{\star}$ (a), $5 R_{\star}$ (b), and $20 R_{\star}$ (c). It is clear that the optical depths are significantly above unity at specific directions even if the injection distance from the massive star is quite large. Since the cross sections for the IC process in the $\mathrm{KN}$ regime and for the $\gamma+\gamma$ collisions $\left(\gamma+\gamma \rightarrow \mathrm{e}^{ \pm}\right)$are comparable, it is expected that relativistic electrons injected isotropically inside the jet lose energy mainly in directions where the optical depths are the largest. They inject primary $\gamma$-rays mainly in the directions of the largest optical depths, i.e., toward the source of soft radiation. These primary $\gamma$-rays can develop IC $\mathrm{e}^{ \pm}$pair cascades in the anisotropic radiation field of the massive star. A part of these cascade $\gamma$-rays escape toward the observer located at specific directions with respect to the binary system. We follow such a complicated cascade process applying the Monte Carlo cascade code described in detail in our previous works (e.g., Bednarek 2000, 2006a). The code also includes the effects connected with synchrotron cooling of the electrons injected into the magnetic field of the jet. Therefore, we are also able to calculate the simultaneous synchrotron photon spectra produced by electrons accelerated in the jet. $\gamma$-ray photons escaping from the binary system are sorted in specific ranges of solid angles on the hemisphere and grouped 

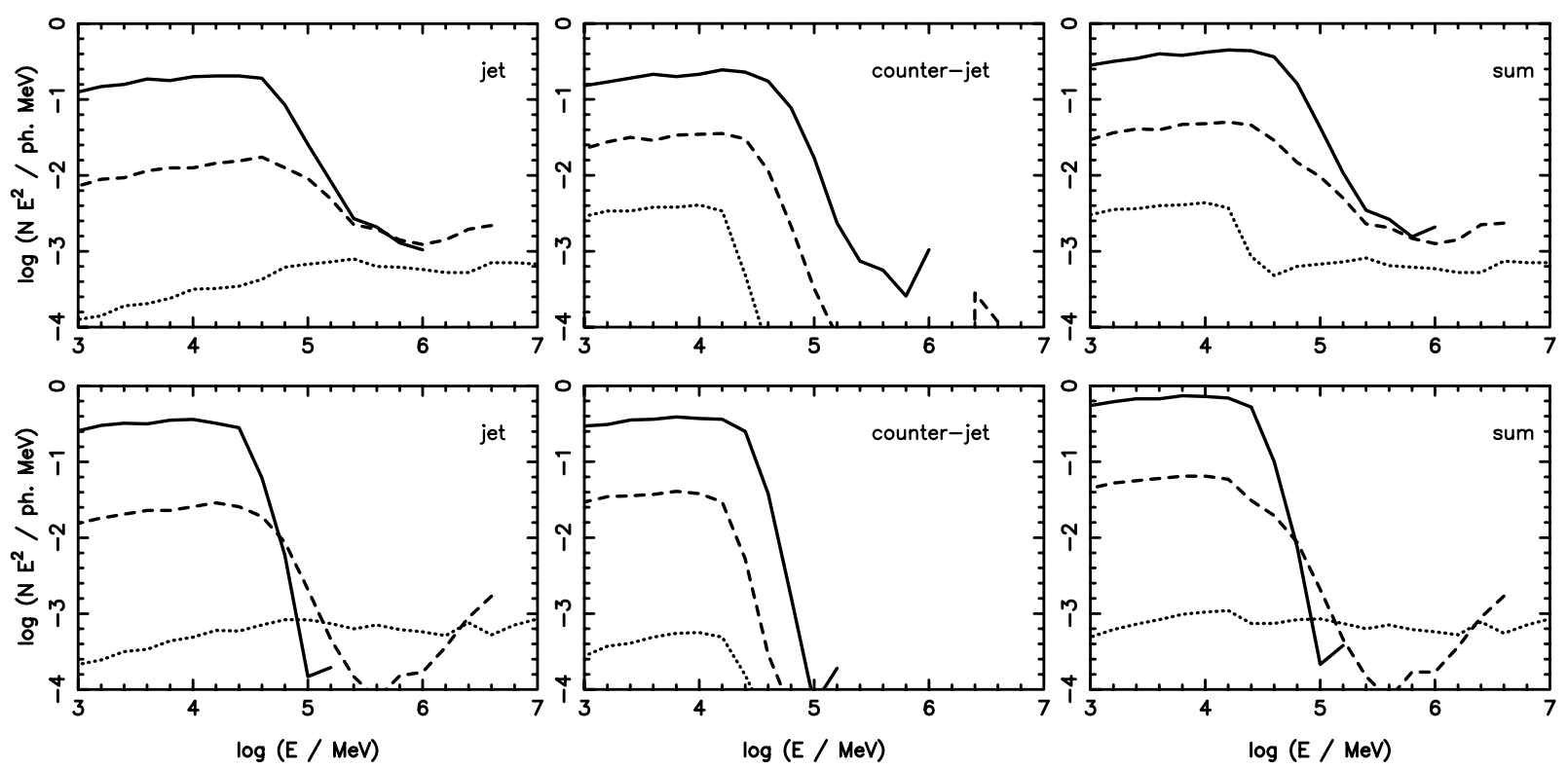

Fig. 3. Cascade $\gamma$-ray spectra produced by electrons injected at different distances from the base of the jet $z=0.1 r_{\star}$ (solid curve), $1 R_{\star}($ dashed), and $10 r_{\star}$ (dotted) for two locations of the compact object on its orbit around the massive star, in front of the massive star (upper figures) and behind the massive star (bottom figures). The $\gamma$-ray spectra expected from the jet moving inside the hemisphere containing the observer, who is located at the inclination angle of $i=30^{\circ}$, are shown on the left figures, from the counter-jet (middle figures), and the sum of the jet + counter-jet spectra (right figures). The acceleration of electrons in the jet is defined by the parameters $\xi=0.1$ and $\eta=0.1$ and the electrons are injected with a power that drops along the jet according to $L_{\mathrm{e}}(z) \propto z^{-2}$.

at specific ranges of energies. Therefore, as a final product, we obtain the $\gamma$-ray spectra produced in such anisotropic cascades at specific directions on the hemisphere.

We note that in parts of the jet relatively close to the accretion disk (this part of the jet is not considered in the present paper), $\gamma$-rays can also be absorbed in the disk radiation field (see, e.g., the calculations of the optical depths by, e.g., Herterich 1974; Protheroe \& Stanev 1987; Carraminana 1992; Bednarek 1993). The disk radiation can be also upscattered to the $\gamma$-ray energies by electrons provided that they are accelerated very close to the base of the jet as considered by, e.g., Aharonian et al. (1985) or Romero et al. (2002).

\section{Cascade gamma-ray spectra}

We investigate the features of the $\gamma$-ray spectra produced in such cascade process as a function of parameters describing the acceleration of electrons in the jet. Since the Doppler factor of the jet is unknown in the case of Cyg X-1 (the lower limit on the jet velocity is $\sim 0.3 \mathrm{c}-$ Fender et al. 2006 - and the inclination angle of the binary system is $i \approx 30^{\circ}$ ), two cases should be considered. When the Doppler factor is low, as e.g., in the case of LS I +61 303 (Bednarek 2006b), then we have to consider the $\gamma$-ray spectra produced not only by electrons in the jet (directed toward the observer), but also the $\gamma$-rays produced by electrons in the counter-jet (directed in the opposite direction). However, for the relativistic jets with Doppler factor $D \gg 1$, it is enough to consider only $\gamma$-ray emission from the observer-directed jet since the contribution from the counter-jet is suppressed due to relativistic effects. Below we study the basic features of the cascade $\gamma$-ray spectra, i.e., their dependence on the distance from the base of the jet, on specific parameters defining the acceleration of electrons, and their dependence on the phase of the binary system and the spectra at specific phases. In the next section we discuss the $\mathrm{GeV}$ and $\mathrm{TeV} \gamma$-ray spectra that might be expected from the massive binary system Cyg X-1.

\subsection{Dependence on the distance from the base of the jet}

Let us at first calculate the $\gamma$-ray spectra escaping from the binary system toward an observer located at the inclination angle $i=30^{\circ}$. These $\gamma$-rays are produced by electrons at different parts of the jet, and their production is parameterized by the distance from the base of the jet. The parameters defining the acceleration of electrons have been chosen from the center of the range of values specified above (i.e., $\xi=0.1$ and $\eta=0.1$, see Fig. 1). For easier interpretation of the results, we apply the differential power-law spectrum of the injected electrons of the type $\propto E^{-2}$ (equal power per decade) up to the maximum energies defined by $\xi$ and $\eta$. In Fig. 3, we show the cascade-produced $\gamma$-ray spectra (multiplied by the energy squared) that are produced by electrons in the jet, the counter-jet, and the sum of both of them, i.e., jet + counter-jet spectra. The upper and lower panels show the spectra that are produced when the compact object is in front of or behind the massive star, respectively. The shape of the $\gamma$-ray spectra clearly depend on the injection distance of electrons.

Electrons that are injected closer to the base of the jet are also closer to the surface of the massive star and therefore are immersed in a stronger soft radiation field. These cascade $\gamma$-ray spectra show a low level of $\mathrm{TeV}$ emission, due to the absorption of $\gamma$-rays, and a relatively high level of $\mathrm{GeV}$ emission (produced due to the efficient cascading). Significant fluxes of $\mathrm{TeV} \gamma$-rays can only be produced in the upper part of the considered jet. The cut-offs in the $\gamma$-ray spectra are determined by the maximum energies of electrons accelerated in the jet. These energies increase with the distance from the base of the jet. The $\gamma$-ray spectra produced toward the observer in the cascade process initiated by electrons in the counter-jet typically show higher $\mathrm{GeV}$ but lower $\mathrm{TeV}$ emission. This is due to the fact that these $\gamma$-rays arrive at the observer after suffering from a severe cascade process (the angle taken from the direction between the jet-counterjet axis and the direction toward the observer is much larger). In fact, electrons in the counter-jet barely contribute to the $\gamma$-ray 

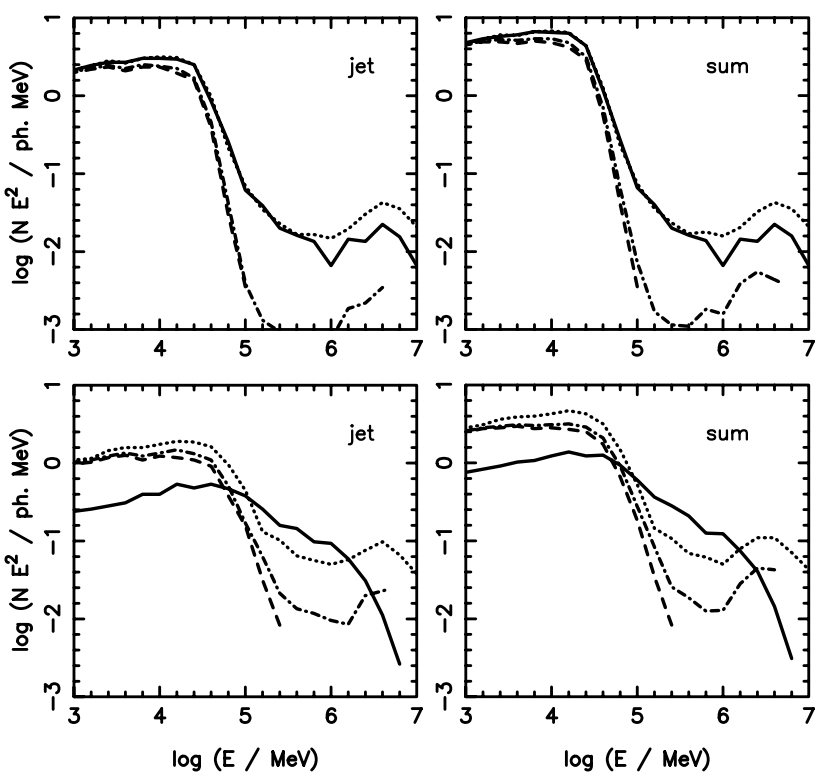

Fig. 4. The cascade $\gamma$-ray spectra (multiplied by energy square) produced by electrons in the jet (left figures) and in the jet + counter-jet (right figures), for the situations when the compact object is behind (upper figures) and in front of the massive star (bottom figures). Specific spectra have been obtained for: $\xi=0.1$ and $\eta=0.1$ (solid curves), $\xi=0.01$ and $\eta=0.1$ (dashed), $\xi=0.1$ and $\eta=0.01$ (dot-dashed), and $\xi=0.3$, and $\eta=0.1$ (dotted). They are integrated over the part of the jet extending between $z=0.1-10 R_{\star}$ measured from the base of the jet. Electrons are injected with the power-law spectrum, $\propto E^{-2}$. The power in electron spectrum drops along the jet according to $L_{\mathrm{e}}(z) \propto z^{-2}$.

spectrum above $\sim 1 \mathrm{TeV}$. Therefore, the sum of the jet + counterjet spectra (right figures) is similar to the spectra from the jet with some increase in the $\mathrm{GeV}$ energy range due to the counterjet contribution. The counter-jet only has the effect of changing the normalization of the total escaping spectra in the GeV energy range.

\subsection{Gamma-ray spectra integrated along the jet}

In Fig. 4, we investigate the dependence of the cascade $\gamma$-ray spectra escaping toward the observer as a function of the parameters defining the acceleration model (see the profiles of the maximum electron energies in Fig. 1). The $\gamma$-ray spectra produced in terms of this model are integrated over the part of the jet extending between $(0.1-10) R_{\star}$, since only from this limited part of the jet can the cascade emission dominate (in the radiation of the massive star) and the $\gamma$-ray emission be correlated with the orbital period of the binary system (as was recently discovered in the case of LS I +61 303, Albert et al. (2006). At distances closer than $0.1 R_{\star}$, the energy losses via synchrotron process and scattering of the disk radiation might dominate if all electrons can be accelerated in that region to above $\sim 1 \mathrm{TeV}$. At distances greater than $10 R_{\star}$, electrons accelerated in the jet are not able to cool locally in the jet. They are advected at large distances along the jet due to the motion of the plasma inside the jet, where they cool via other processes. Note, also, that scenarios in which the possible production of $\gamma$-rays occurs very close or far away from the base of the jet do not provide an explanation of the correlation of the $\gamma$-ray emission with the period of the binary system.

From a comparison of calculations performed for different parameters, we conclude that strong $\mathrm{TeV} \gamma$-ray emission appears for larger values of the acceleration efficiency of electrons in the jet (see Fig. 4). As expected, the $\gamma$-ray spectra generally cut off at lower energies for the case of less magnetized jets and lower acceleration parameters. This is due to the saturation of electron acceleration at lower energies by IC energy losses in these cases (see Fig. 1). The $\gamma$-ray spectra produced in the cascade initiated by electrons, when the jet is behind the massive star, show a sharp cut off at $\sim 100 \mathrm{GeV}$ due to the efficient cascading for all the considered cases. However, when the compact object is in front of the massive star, the $\mathrm{TeV} \gamma$-ray emission can reach the observer with a relatively flat spectrum (spectral index $\sim 2.0-2.5$ above a few $100 \mathrm{GeV}$ ) and the flux at a level of $\sim 0.1-0.01$ of the $\mathrm{GeV}$ emission. We conclude that the $\gamma$-ray emission produced by electrons in the jet in terms of the considered model has very interesting features that should allow us to explicitly distinguish between different models for the $\gamma$-ray production in massive binaries, e.g., scattering of the disk radiation by electrons in the jet (as considered in the case of active galaxies by, e.g., Dermer $\&$ Schlickeiser 1993) or production of $\gamma$-rays far away from the base of the jet (e.g., Atoyan \& Aharonian 1999).

\subsection{Gamma-ray light curve and phase-dependent spectra}

Let us now discuss the dependence of $\gamma$-ray emission as a function of the phase of the binary system, where the zero phase is taken when the location of the compact object is behind the massive star. Since the orbit of the compact object is almost circular, the $\gamma$-ray light curves should be symmetrical. We calculate the number of $\gamma$-ray photons that escape from the binary system toward the observer located at the inclination angle $i=30^{\circ}$, in two energy ranges: the so-called GeV energy range (between 1-10 GeV available for the past EGRET telescope and the future AGILE and GLAST telescopes), and the TeV energy range ( $>200 \mathrm{GeV})$ available for the present and future Cherenkov telescopes on the northern hemisphere such as the MAGIC and VERITAS telescopes. As an example, we concentrate on the model defined by $\xi=0.1$ and $\eta=0.1$ (the parameters from the center of considered range), and the electron spectrum described by $\propto E^{-2}$, and its power dropping along the jet according to $L_{\mathrm{e}}(z) \propto z^{-2}$. The $\gamma$-ray light curves (number of photons arriving to the observer in the $\mathrm{GeV}$ and $\mathrm{TeV}$ energy ranges) are shown in Fig. 5 for the case of $\gamma$-ray emission only from the jet and the sum of the emission from the jet + counterjet. The TeV $\gamma$-ray emission can change in this case by more than a factor of 20, showing the minimum when the compact object is behind the massive star and a sharp maximum for the phases at $\sim 0.05$ before and after the situation when the compact object is in front of the massive star.

Exactly in front of the massive star, the $\mathrm{TeV} \gamma$-ray flux becomes a factor of $\sim 2$ lower. Due to the lowest optical depths for $\gamma$-rays and leptons, production of $\gamma$-rays is less favored in such a geometry than in surrounding phases. The contribution of the counter jet to the maximum in the $\mathrm{TeV} \gamma$-ray light curve is at the level of $\sim 30 \%$ of the jet emission (compare the curves). On the other hand, the $\mathrm{GeV} \gamma$-ray light curve behaves differently. It reaches its maximum when the compact object is behind the massive star and its minimum exactly when the compact object is in front of the massive star. The maximum is due to the fact that the $\gamma$-ray spectrum is formed in the very efficient cascade process when the compact object and the observer are on opposite sites of the massive star. The contributions of the jet and the counter-jet to the GeV $\gamma$-ray light curve are comparable. It is remarkable that there is a very interesting anticorrelation between the $\gamma$-ray light curves in the $\mathrm{GeV}$ and $\mathrm{TeV}$ energy ranges. This 

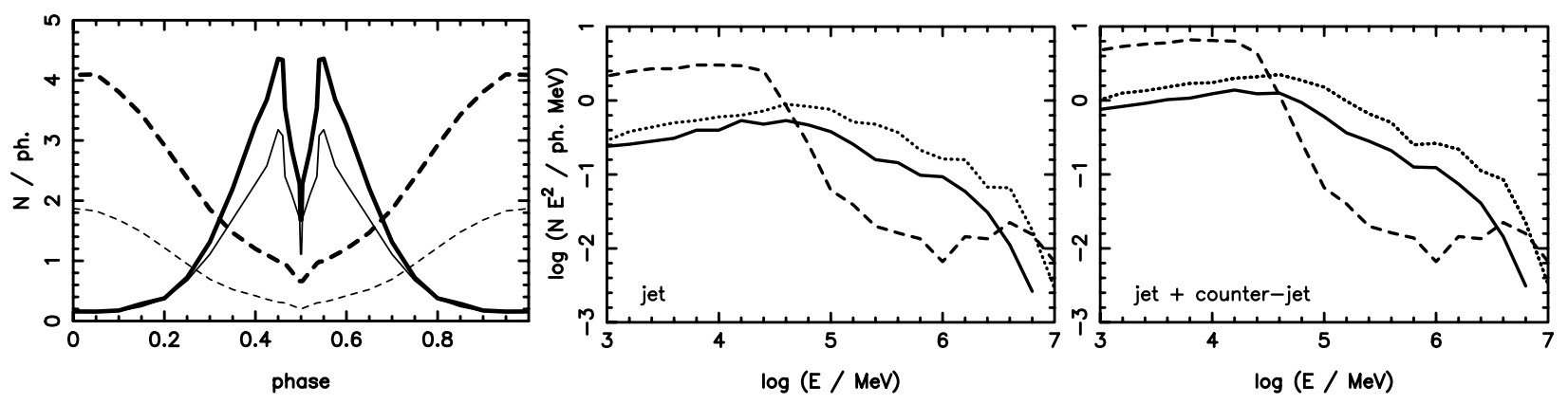

Fig. 5. Gamma-ray light curves in the energy range $1-10 \mathrm{GeV}$ (dashed curves) and $>200 \mathrm{GeV}$ (solid curves) produced by electrons accelerated in the jets of Cyg X-1 for the model defined by the parameters $\xi=0.1$ and $\eta=0.1$, the power-law spectrum of electrons, $\propto E^{-2}$, and the power transferred to electrons from the acceleration mechanism that drops along the jet according to $L_{\mathrm{e}}(z) \propto z^{-2} \cdot \gamma$-rays produced by electrons in the jet (thin curves) and in the jet + counter-jet (thick curves). The middle and right panels show the cascade IC $\gamma$-ray spectra escaping to the observer from the binary system for three phases of the compact object (measured from the location of the compact object behind the massive star): 0.0 (dashed curve), 0.45 (dotted), and 0.5 (solid).

is the unique feature that can serve as an important diagnostic of this model for the high-energy processes occurring in the $\gamma$-ray emitting massive binary systems.

Figure 5 also shows the $\gamma$-ray spectra emerging toward the observer for some characteristic phases of the binary system for the case of the acceleration of electrons in the jet and the sum for the jet + counter-jet emission. Note the dependence between the spectral shape and the flux of the $\mathrm{TeV} \gamma$-ray emission. It is predicted that the lower level of $\gamma$-ray flux should be connected with a flattening of the spectrum above $\sim 200 \mathrm{GeV}$ and vice versa. The contribution of $\gamma$-ray emission from the counter-jet has an effect of increasing the GeV $\gamma$-ray flux with no effect on the TeV flux. However, due to the higher $\mathrm{GeV}$ emission, the relative power in the $\mathrm{GeV}$ and $\mathrm{TeV}$ energy ranges changes. This has consequences for predicting the TeV flux from the normalization to the $\mathrm{GeV}$ (EGRET) observations.

\section{The multifrequency spectrum of Cyg X-1}

The $\gamma$-ray spectrum of Cyg X-1 is in fact very poorly constrained. The spectrum in the soft X-ray state shows the powerlaw tail with the differential spectral index -2.6 extending up to $\sim 10 \mathrm{MeV}$, without evidence of a cut off (McConnell et al. 2000, 2002). However, the jet appears only in the low-hard state, so this emission can be only considered as an upper limit on the $\mathrm{MeV} \gamma$-ray flux related to the jet. The upper limit at higher energies (>100 MeV) has been derived (see McConnell et al. 2000), based on the 3rd EGRET catalog (Hartman et al. 1999).

However, in the context of the recent discoveries of the $\mathrm{TeV}$ emission from massive binary systems (i.e., LS 5039 and LS I +61 303), which have similar orbital parameters to Cyg X-1 (i.e., a massive star and separation of the components), it is reasonable to predict the level and spectral features of the possible high energy $\gamma$-ray emission from this object also. Based on the IC $\mathrm{e}^{ \pm}$pair cascade model (Bednarek 2006b), considered here for Cyg X-1, we were able to predict the phase dependent TeV emission from LS I +61 303 (Albert et al. 2006). In the case of Cyg X-1, we normalize the cascade $\gamma$-ray spectra expected from this object to the upper limit derived from the EGRET data. Note that the $\mathrm{MeV} \gamma$-ray emission from Cyg X-1 is $\sim 1 \%$ of its total bolometric power (McConnell et al. 2002), and the EGRET upper limit in the GeV energies is again on the level of $10 \%$ of the $\mathrm{MeV}$ emission. On the other hand, in the cascade model, we consider that almost all energy from relativistic electrons in the jet is transfered to gamma-rays (and also secondary pairs), see
Bednarek (2006b). Since the jet power is expected to be comparable to the observed luminosity of the Cyg X-1, we conclude that the considered model is consistent if only a small part of jet energy is converted into relativistic electrons (i.e., $\sim 0.1 \%$ ). The applied normalization to the EGRET upper limit allows us to estimate the level of the $\mathrm{TeV} \gamma$-ray flux and spectrum from Cyg X-1. In Fig. 6, we show the comparison of the results of calculations with the observational constraints at lower energies. In the upper and the lower panels, we show the cascade $\gamma$-ray spectra escaping toward the observer located at the inclination angle $i=30^{\circ}$ for the case of their production in the jet and the jet+counter-jet (the sum of both), respectively. Since we have no detailed information about the spectral shape in the GeV energy range from the EGRET observations (except the upper limit), electrons injected into the jet with different spectra and injection efficiencies along the jet have to be considered. At first we consider the model with very flat electron spectrum (differential spectral index equal to -2) and the power transferred to electrons which changes with distance along the jet according to $L_{\mathrm{e}}(z) \propto z^{-2}$ (model A). In fact, such a flat electron spectrum seems to be not very realistic if we compare the spectral features of the source LS I +61 303 (the object of our previous modeling) and the spectrum of Cyg X-1. The electron spectrum with the index 2 postulates the appearance of a completely new component in the high energy spectrum of Cyg X-1, which does not lie on the extrapolation from the $\mathrm{MeV}$ energy range. The comparison of the calculated cascade spectra with the available data and the sensitivities of the present MAGIC telescope (M) and the future VERITAS telescope (V) is shown in Figs. 6a,b. The curves show the expected spectra when the compact object is in front of and behind the massive star HDE 226868. Clearly, for such model parameters, Cyg X-1 can potentially be detected at energies above $\sim 100 \mathrm{GeV}$ by the MAGIC telescope and above a few $100 \mathrm{GeV}$ by the VERITAS telescope, when the compact object is in front of the massive star. We also show the synchrotron spectrum, which is produced by electrons accelerated in the jets, assuming that the Doppler boosting is not important (marked bumps at lower energies). These spectra are at a much lower level than the observations of Cyg X-1 in the MeV energy range and cannot be responsible for this emission. Note, however, that if significant Doppler boosting is discovered in Cyg X-1 (the speed of the jet comparable to the velocity of light), the flux in the calculated synchrotron spectrum should go up with respect to the $\gamma$-ray spectra. This is due to the fact that the cascade $\gamma$-ray spectra are mainly formed outside the jet, i.e., inside the whole 

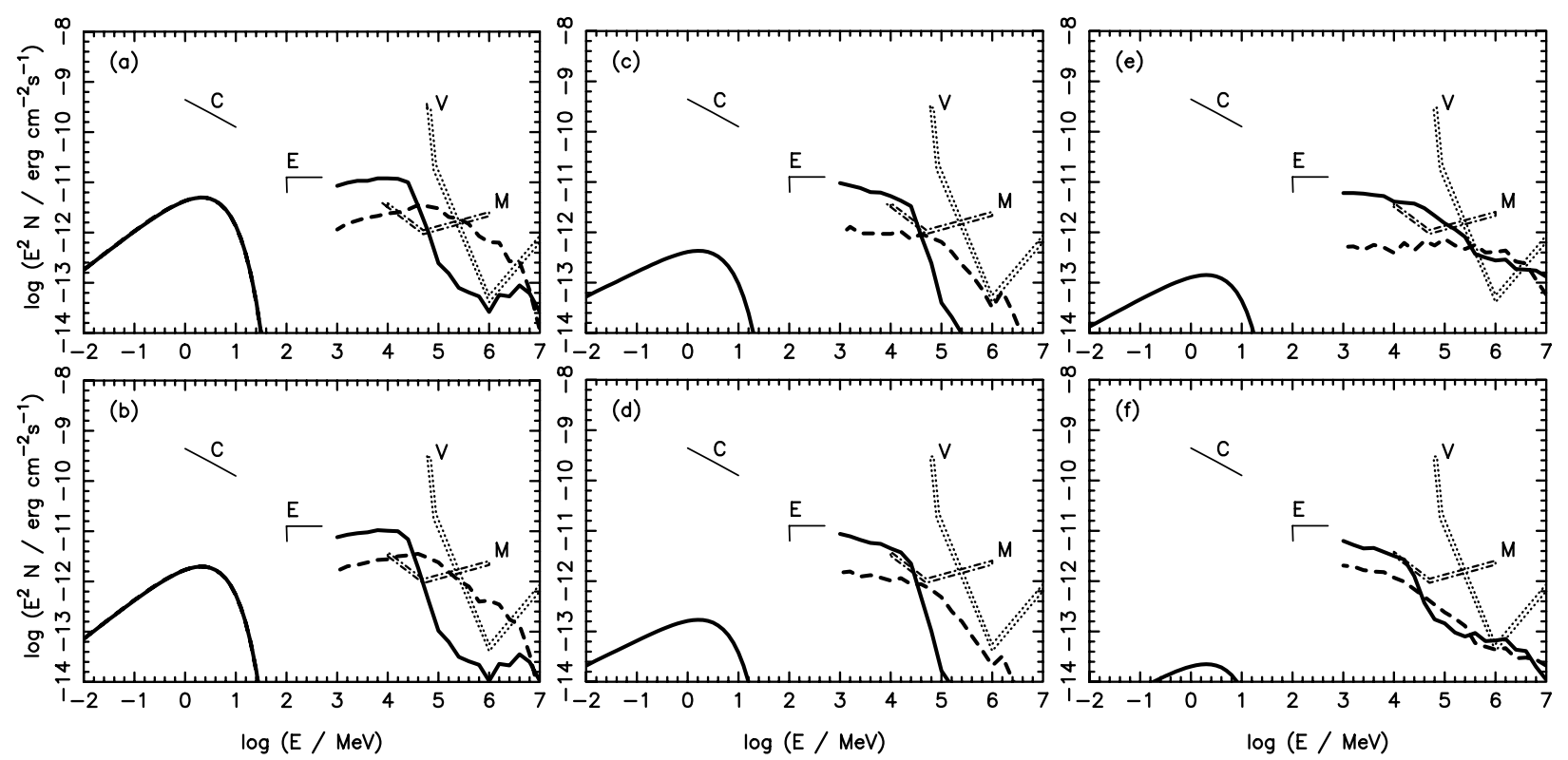

Fig. 6. The multiwavelength spectrum of Cyg X-1 (differential spectrum multiplied by energy squared) is compared with the calculated cascade $\gamma$-ray spectra for three acceleration models of electrons in the jet: (1) differential spectral index of electrons $\propto E^{-\alpha}$ and $\alpha=2$, and the power transfered to electrons changes with distance along the jet according to $L_{\mathrm{e}}(z) \propto z^{-2}$ (model A); (2) $\alpha=2.6$ and $L_{\mathrm{e}}(z) \propto z^{-2}$ (model B); and (3) $\alpha=$ 2.6 and $L_{\mathrm{e}}(z)=$ const. (model $\mathrm{C}$ ). The cut-off in the electron spectrum is determined by $\xi=0.1, \eta=0.1$. The upper and lower panels show the spectra produced in the jet and the jet + counter-jet, respectively. The $\gamma$-ray spectra above are shown for the minimum flux above $100 \mathrm{GeV}$, i.e., at the phase 0.0 counted from the location of the compact object behind the massive star (solid curves), and for the maximum flux at the phase $\sim 0.45$ and 0.55 (dashed curves). The synchrotron spectrum produced by electrons in the jet are shown by the solid curves below MeV energies. The spectrum from Cyg X-1 reported by the COMPTEL telescope and the upper limit based on the EGRET data (McConnell et al. 2000, 2002) are shown by thin solid curves and marked by $\mathrm{C}$ and $\mathrm{E}$, respectively. The sensitivities of the MAGIC and VERITAS telescopes are shown by double dot-dashed and dotted curves and marked by M and V, respectively (see, e.g., Lorenz 2000).

volume of the binary system where the plasma moves with the wind velocity of the massive star. In such a case, the Doppler boosting can be only important for the synchrotron radiation, but is clearly unimportant for cascade $\gamma$-rays. This $\mathrm{MeV} \gamma$-ray emission can also originate in another scenario, e.g., in the hot corona of the accretion disk (e.g., Zdziarski \& Gierliński 2004; Chakrabarti \& Mandal 2006) or in the inner part of the jet close to its base (a scenario not considered in this paper) as a result of Comptonization of the disk radiation and possible IC cascading in the disk radiation, e.g., Aharonian et al. (1985); Coppi et al. (1993).

The calculations with the more realistic model parameters (see Table 2) are compared with the Cyg X-1 data and sensitivities of the Cherenkov telescopes and are shown in Fig. 6. We show the $\gamma$-ray spectra from the jet in Fig. 6c and the jet + counter-jet, which are calculated for electrons with the differential spectral index $\propto E^{-2.6}$, and the power in electrons dropping along the jet according to $L_{\mathrm{e}}(z) \propto z^{-2}$ (model B) in Figs. 6d. For these parameters, there is no chance for the detection of TeV $\gamma$-ray emission either by the MAGIC telescope above $\sim 100 \mathrm{GeV}$ or by the Veritas telescope at $\sim 1 \mathrm{TeV}$. Finally in Figs. 6e, f, the $\gamma$-ray spectra are shown for electrons injected with the steep spectrum $\left(\propto E^{-2.6}\right)$, but with a flat profile for the power transferred from the acceleration mechanism to electrons, $L_{\mathrm{e}}(z)=$ const. (model C). For this model, a large amount of power is transferred to electrons at larger distances from the base of the jet where the acceleration of electrons is saturated at larger energies. Then, the cascade $\gamma$-ray spectra at $\mathrm{TeV}$ energies are relatively flat, in spite of the strong $\gamma$-ray absorption effects. The $\gamma$-ray spectra produced from the jet, after normalization to the EGRET upper limit in the GeV energy range, have a chance to be detected by the VERITAS telescope at TeV energies, but not
Table 2. Parameters of the models considered in Fig. 6.

\begin{tabular}{cccc}
\hline \hline Model & $\mathrm{A}$ & $\mathrm{B}$ & $\mathrm{C}$ \\
\hline $\begin{array}{c}\text { differential spectrum } \\
\text { of electrons: } N(E) \propto\end{array}$ & $E^{-2}$ & $E^{-2.6}$ & $E^{-2.6}$ \\
\hline $\begin{array}{c}\text { injection number of electrons } \\
\text { along the jet: } L_{\mathrm{e}}(z) \propto\end{array}$ & $z^{-2}$ & $z^{-2}$ & const. \\
\hline
\end{tabular}

by the MAGIC telescope above $\sim 100 \mathrm{GeV}$ (Fig. 6e). Note that the unboosted synchrotron spectra calculated in models $\mathrm{B}$ and $\mathrm{C}$ are at a lower level than in model A, due to different normalization in the $\mathrm{GeV}$ energy range.

\section{Conclusions}

We constrain the parameters of the IC $\mathrm{e}^{ \pm}$pair cascade model for which we predict observable $\gamma$-ray fluxes from Cyg X-1 at energies $>100 \mathrm{GeV}$ by the MAGIC and/or VERITAS telescopes. The main conclusions from the analysis of this model for the $\gamma$-ray production in microquasars are reported in the following. The detection of the $\mathrm{TeV} \gamma$-ray emission from Cyg X-1 by Cherenkov telescopes can occur only under very special conditions for the acceleration of electrons in the jet as described in the model herein considered, and for an acceleration efficiency parameter $\xi$, the magnetic field strength parameter $\eta$, a flat spectrum of electrons, and a flat profile of the conversion of energy from the acceleration mechanism to electrons along the jet. These conditions are not very likely and do not fit nicely to $\gamma$-ray observations of Cyg X-1 at the $\mathrm{MeV}-\mathrm{GeV}$ energy range.

Provided that such strict conditions for the acceleration of electrons are met (e.g., as in the considered model A), we have 
found that in the case of compact massive binary systems with circular orbits of the Cyg X-1 type, the highest chances for detection of $\mathrm{TeV} \gamma$-ray emission by the Cherenkov telescopes are for phases $\sim 0.45$ and $\sim 0.55$, counted from the position of the compact object behind the massive star. The flux expected at the moment when the compact object is exactly in front of the massive star is a factor of $\sim 2$ lower. The $\mathrm{GeV}$ and $\mathrm{TeV} \gamma$-ray fluxes should be anticorrelated. The largest GeV flux is expected when the compact object is behind the massive star. The GeV $\gamma$-ray flux should vary by a factor of $\sim 5$ and the TeV flux by a factor of $\sim 20$ with the orbital period of Cyg X-1/HDE 226868 system.

We want to remind the reader that the above conclusions concerning the possible detection of the very high energy $\gamma$-ray emission from Cyg X-1 are based on the assumption that the upper limit derived from the EGRET observations provides correct constraints on the multifrequency spectrum of Cyg X-1, independent of the activity state of this binary system. In fact, this upper limit is consistent with the extrapolation of the $\gamma$-ray spectrum reported by the COMPTEL telescope from the Cyg X-1 region during the hard X-ray state of this source. Such a flat $\mathrm{MeV}$ spectrum is not observed in Cyg X-1 during its other activity states (e.g., McConnell et al. 2000, 2002).

Acknowledgements. We thank the anonymous referee for valuable comments and James H. Beall for reading the manuscript and providing comments. This work is supported by the Polish MNiI grant No. 1P03D01028.

\section{References}

Aharonian, F., Kirillov-Ugryumov, V. G., \& Vardanian, V. V. 1985, Ap\&SS, 115, 201

Aharonian, F., Akhperjanian, A. G., Aye, K.-M., et al. 2005a, A\&A, 442, 1 Aharonian, F., Akhperjanian, A. G., Aye, K.-M., et al. 2005b, Science, 309, 746 Aharonian, F., Akhperjanian, A. G., Aye, K.-M., et al. 2006a, A\&A, 460, 743 Aharonian, F., Anchordoqui, L. A., Khangulyan, D., \& Montaruli, T. 2006b, J. Phys. Conf. Ser., 39, 408

Albert, J., Aliu, E., Anderhub, H., et al. 2006, Science, 312, 1771

Atoyan, A. M., \& Aharonian, F. 1999, MNRAS, 302, 253

Atoyan, A. M., Aye, K.-M., Chadwick, P. M., et al. 2002, A\&A, 383, 864

Bednarek, W. 1993, A\&A, 278, 307

Bednarek, W. 1997, A\&A, 322, 523

Bednarek, W. 2000, A\&A, 362, 646

Bednarek, W. 2005, ApJ, 631, 466

Bednarek, W. 2006a, MNRAS, 368, 579
Bednarek, W. 2006b, MNRAS, 371, 1737

Bednarek, W., \& Giovannelli, F. 1999, Mem.SAIt, 70, 1071

Bednarek, W., Giovannelli, F., Karakuła, S., \& Tkaczyk, W. 1990, A\&A, 236, 268

Blumenthal, G. R., \& Gould, R. J. 1970, Rev. Mod. Phys., 42, 237

Bolton, C. T. 1972, Nature, 235, 271

Bosch-Ramon, V., Romero, G. E., \& Paredes, J. M. 2005, A\&A, 429, 267

Bosch-Ramon, V., Romero, G. E., \& Paredes, J. M. 2006, A\&A, 447, 263

Bowyer, S., Byram, E. T., Chubb, T. A., \& Friedman, H. 1965, Science, 147, 394

Böttcher, M., \& Dermer, C. D. 2005, ApJ, 634, 81

Braes, L. L. E., \& Miley, G. K. 1971, Nature, 232, 246

Carraminana, A. 1992, A\&A, 264, 127

Chadwick, P. M., Daniel, M. K., Lyons, K., et al. 2000, A\&A, 364, 165

Chakrabarti, S. K., \& Mandal, S. 2006, ApJ, 642, L49

Coppi, P., Blandford, R. D., \& Rees, M. J. 1993, MNRAS, 262, 603

Dermer, C. D., \& Böttcher, M. 2006, ApJ, 643, 1081

Dermer, C. D., \& Schlickeiser, R. 1993, ApJ, 416, 458

Dubus, G. 2006, A\&A, 451, 9

Falcke, H., Malkan, M. A., \& Biermann, P. L. 1995, A\&A, 298, 375

Falcke, H., Körding, E., \& Markoff, S. 2004, A\&A, 414, 895

Fender, R. P., Stirling, A. M., Spencer, R. E., et al. 2006, MNRAS, 369, 603

Gallo, E., Fender, R., Kaiser, C., et al. 2005, Nature, 436, 819

Georganopoulos, M., Aharonian, F. A., \& Kirk, J. G. 2002, A\&A, 388, L25

Gierliński, M., Zdziarski, A. A., Poutanen, J., et al. 1999, MNRAS, 309, 496

Hartman, R. C., et al. 1999, ApJS, 123, 79

Herterich, K. 1974, Nature, 250, 311

Hjellming, R. M., \& Wade, C. M. 1971, ApJ, 168, L21

Kniffen, D. A., Alberts, W. C. K., Bertsch, D. L., et al. 1997, ApJ, 486, 126

Liang, J. C., \& Nolan, P. L. 1983, Space Sci. Rev., 38, 353

Ling, J. C., Mahoney, W. A., Wheaton, W. A., Jacobsen, A. S., \& Kaluzienski, L. 1983, ApJ, 275, 307

Lorenz, E. 2000, J. Phys. G, 27, 1675

McConnell, M. L., Ryan, J. M., Collmar, W., et al. 2000, ApJ, 543, 928

McConnell, M. L., Zdziarski, A. A., Bennett, K., et al. 2002, ApJ, 572, 984

Mori, M., Bertsch, D. L., Dingus, B. L., et al. 1997, ApJ, 476, 842

Paredes, J. M., Marti, J., Ribo, M., \& Massi, M. 2000, Science, 288, 2340

Poutanen, J., Krolik, J. H., \& Ryde, F. 1997, MNRAS, 292, L21

Priedhorsky, W. C., Terrell, J., \& Holt, S. S. 1983, ApJ, 270, 233

Protheroe, R. J., \& Stanev, T. 1987, ApJ, 322, 838

Romero, G. E., Kaufman Bernado, M. M., \& Mirabel, I. F. 2002, A\&A, 393, L61

Romero, G. E., Christiansen, H. R., \& Orellana, M. 2005, ApJ, 632, 1093

Stirling, A. M., Spencer, R. E., de la Force, C. J., et al. 2001, MNRAS, 327, 1273

Thompson, D. J., Bertsch, D. L., Dingus, B. L., et al. 1995, ApJS, 101, 259

Vestrand, W. T., Sreekumar, P., \& Mori, M. 1997, ApJ, 483, L49

Webster, B. L., \& Murdin, P. 1972, Nature, 235, 37

Weekes, T. C. 1988, Phys. Rep., 160, 1

Weekes, T. C. 1992, SSRv, 59, 315

Zdziarski, A. A., \& Gierliński, M. 2004, Prog. Theor. Phys. Suppl., 155, 99

Ziółkowski, J. 2005, MNRAS, 358, 851 